Article

\title{
Connexin 43 Gene Therapy Delivered by Polymer-Modified Salmonella in Murine Tumor Models
}

\author{
Wei-Kuang Wang ${ }^{1}$, Yu-Diao Kuan ${ }^{2}$, Chun-Yu Kuo ${ }^{3}$ and Che-Hsin Lee ${ }^{2,3, *}$
}

1 Department of Environmental Engineering and Science, Feng Chia University, Taichung 407, Taiwan; E-Mail: wkwang@fcu.edu.tw

2 Graduate Institute of Basic Medical Science, School of Medicine, China Medical University, Taichung 404, Taiwan; E-Mail: yoite_to_miharu@hotmail.com

3 Department of Microbiology, School of Medicine, China Medical University, Taichung 404, Taiwan; E-Mail: susan110272@hotmail.com

* Author to whom correspondence should be addressed; E-Mail: chlee@mail.cmu.edu.tw; Tel.: +886-4-2205-3366 (ext. 2173); Fax: +886-4-2205-3764.

Received: 29 January 2014; in revised form: 11 March 2014 / Accepted: 31 March 2014 / Published: 11 April 2014

\begin{abstract}
The use of preferentially tumor-targeting bacteria as vectors is one of the most innovative approaches for the treatment of cancer. This method is based on the observation that some obligate or facultative anaerobic bacteria are capable of selectively multiplying in tumors and inhibiting their growth. Previously, we found that the tumor-targeting efficiency of Salmonella could be modulated by modifying the immune response to these bacteria by coating them with poly(allylamine hydrochloride) (PAH), and these organisms are designated PAH-S.C. (S. choleraesuis). PAH can provide a useful platform for the chemical modification of Salmonella, perhaps by allowing a therapeutic gene to bind to tumor-targeting Salmonella. This study aimed to investigate the benefits of the use of PAH-S.C. for gene delivery. To evaluate this modulation, the invasion activity and gene transfer of DNA-PAH-S.C. were measured in vitro and in vivo. Treatment with PAH-S.C. carrying a tumor suppressor gene (connexin 43) resulted in inhibition of tumor growth, which suggested that tumor-targeted gene therapy using PAH-S.C. carrying a therapeutic gene could exert antitumor activities. This technique represents a promising strategy for the treatment of tumors.
\end{abstract}

Keywords: Salmonella; poly(allylamine hydrochloride); tumor-targeting; connexin 43 


\section{Introduction}

Successful cancer gene therapy relies on the development of vectors that can selectively and effectively deliver a therapeutic gene to tumor sites. Salmonella are Gram-negative, facultative anaerobes that are a common cause of intestinal infections. Salmonella grow under aerobic and anaerobic conditions; thus, they are able to colonize small metastatic and larger tumors. Attenuated Salmonella have been shown to inhibit tumor growth in a broad range of human and mouse tumors [1-5], and Salmonella-based vectors have been considered potential antitumor agents for tumor vaccines, gene delivery, and tumor-targeting vectors [6-8]. Purified proteins, plasmids, and viruses can be encapsulated in a biodegradable polymer and delivered through various routes [9], and the interactions of polymers with biological systems have been a topic of interest in widely divergent fields. Living cells can be encapsulated by the alternating adsorption of oppositely charged polyelectrolytes, and the metabolic activity of the coated cells is well preserved after encapsulation [10]. This study demonstrates that the masking of Salmonella with a polymer and DNA results in the tumor-targeting gene transfer of Salmonella following in vivo delivery. We tested this exogenous, tumor-specific gene delivery into tumors using Salmonella to increase therapeutic gene expression and delay tumor growth.

\section{Experimental Section}

\subsection{Cells, Bacteria, Reagents, and Mice}

Mammary carcinoma cells (4T1) were cultured in Dulbecco's modified Eagle's medium (DMEM, Hyclone, Logan, UT, USA) supplemented with $50 \mu \mathrm{g} / \mathrm{mL}$ gentamicin, $2 \mathrm{mM}$ L-glutamine, and 10\% heat-inactivated fetal bovine serum (FBS) at $37{ }^{\circ} \mathrm{C}$ in $5 \% \mathrm{CO}_{2}$ [11]. A vaccine strain of Salmonella enterica serovar Choleraesuis (ATCC 15480) was obtained from the Bioresources Collection and Research Center (Hsinchu, Taiwan). This rough variant of S. choleraesuis (S.C.), designated vaccine 51, was obtained by spreading an 18-h broth culture of the virulent strain 188 of $S$. choleraesuis strain Dublin over the surface of a dried nutrient agar plate, placing a drop of a suspension of Salmonella anti-O phage No. 1, and selecting for a phage-resistant colony after incubation at $37{ }^{\circ} \mathrm{C}$ for $24 \mathrm{~h}$ [12]. Poly(allylamine hydrochloride) (PAH; $\left.M_{\mathrm{W}}: 15,000\right)$ and 4',6-diamidino-2-phenylindole (DAPI) were purchased from Sigma-Aldrich (St. Louis, MO, USA). Female BALB/c mice (6- to 8-weeks-old) were obtained from the National Laboratory Animal Center of Taiwan and maintained in a specialized pathogen-free animal care facility in isothermal conditions with regular photoperiods. The experimental protocol adhered to the rules of the Animal Protection Act of Taiwan and was approved by the Laboratory Animal Care and Use Committee of the China Medical University (permit number: 103-49-N).

\subsection{Preparation of DNA-PAH-Modified Salmonella}

S.C. $\left(10^{6}\right.$ colony-forming units (cfu)) were washed with deionized water to remove nutrients and metabolites from the bacteria. The washed S.C. were resuspended in $1 \mathrm{~mL}$ of PAH solution ( $5 \mathrm{mg} / \mathrm{mL})$, incubated on a shaker for $15 \mathrm{~min}$, and then centrifuged (10 min at $1000 \times \mathrm{g})$ [12]. The excess PAH solution was discarded, and the S.C. were dispersed and washed three times in water. 
The PAH-S.C. $\left(10^{6} \mathrm{cfu}\right)$ were incubated with $5 \mu \mathrm{g}$ of plasmid DNA (pTCY-EGFP, pTCY-Luc or pcDNA3.1-connexin $43(\mathrm{Cx} 43))$ on the shaker for $30 \mathrm{~min}$ and then centrifuged $(10 \mathrm{~min}$ at $1000 \times \mathrm{g})$. The supernatant solution was discarded, and the DNA-PAH-S.C. were dissolved in $1 \mathrm{~mL}$ PBS.

\subsection{Characterization of the DNA-PAH-Modified Salmonella}

The size distributions and zeta potential values of the S.C., PAH-modified S.C. (PAH-S.C.), and DNA-PAH-modified S.C. were measured in deionized water using a dynamic light scattering system (Zetasizer ZS90, Malvern instruments, Malvern, UK) [12].

\subsection{Animal Studies}

Groups of mice were subcutaneously (s.c.) inoculated with $10^{6}$ tumor cells. When the tumors had grown to diameters between 50 and $100 \mathrm{~mm}^{3}$, the mice were intravenously (i.v.) injected with PBS, Luc-PAH-S.C., or Cx43-PAH-S.C. These groups of mice were sacrificed at various time points post-infection, and the numbers of Salmonella in the tumors, livers, and spleens were determined on LB agar plates; these data were expressed as cfu per gram of tissue. Tissue homogenates were also assessed for luciferase activity using a luciferase reporter gene assay system (Promega, Madison, WI, USA). In a separate experiment, palpable tumors were measured every 3 days in two perpendicular axes using a tissue caliper, and the tumor volumes were calculated as follows: (length of tumor) $\times$ (width of tumor) ${ }^{2} \times 0.45$. The survival rates of the mice in the treated and control groups were monitored daily [11].

\subsection{Infection of Tumor Cells with Salmonella}

EGFP-PAH-S.C. or Luc-PAH S.C. $\left(10^{6} \mathrm{cfu}\right)$ were incubated with $4 \mathrm{~T} 1$ cells $\left(10^{5}\right.$ cells/well) for $8 \mathrm{~h}$. The medium was then removed, the cells were washed, and fresh medium supplemented with $50 \mu \mathrm{g} / \mathrm{mL}$ gentamicin was added. The supernatants were removed after an additional $90 \mathrm{~min}$ of culture in gentamicin, and adherent cells were lysed to release the intracellular bacteria. Cells stained with DAPI were detected using a fluorescence microscope at a magnification of $\times 200$. In a separate experiment, Luc-PAH-S.C. was added to the cells, which were then cultured in $1 \mathrm{~mL}$ of antibiotic-free medium and incubated for $8 \mathrm{~h}$. All of the cells were washed, replenished with complete medium containing gentamicin $(50 \mu \mathrm{g} / \mathrm{mL})$, and cultured for $16 \mathrm{~h}$. The cells were lysed to prepare extracts for the determination of luciferase activity using a luciferase assay kit (Promega) [13].

\subsection{Western Blot Analysis}

Tumor lysates were prepared by extracting proteins with lysis buffer at day 10, and cytoplasmic and nuclear fractions were prepared according to the manufacturer's instructions (Pierce Biotechnology, Rockford, IL, USA). Proteins from total cell extracts were fractionated using SDS-PAGE, transferred onto Hybond enhanced chemiluminescence nitrocellulose membranes (Amersham, Little Chalfont, UK), and probed with primary antibodies against connexin 43 (AC-15, Sigma-Aldrich) or monoclonal antibodies against $\beta$-actin (AC-15, Sigma-Aldrich). Horseradish peroxidase-conjugated secondary antibodies were used, and protein-antibody complexes were visualized using an enhanced 
chemiluminescence system (Amersham) [14]. The signals were quantified using the ImageJ software (rsbweb.nih.gov/ij/) [15-17].

\subsection{Statistical Analysis}

All data are expressed as the mean \pm standard deviation (SD). An unpaired, two-tailed Student's $t$-test was used to determine differences between groups. A $p$ value less than 0.05 was considered statistically significant.

\section{Results and Discussion}

\subsection{Characterization of Poly(allylamine hydrochloride) (PAH)-Modified Salmonella}

The bacterial cell wall is negatively charged due to the presence of teichoic acid in Gram-positive bacteria or lipopolysaccharide (LPS) in Gram-negative bacteria. Positively charged polyelectrolytes can bind to negatively charged bacterial surfaces to form thin films that can attach to negatively charged materials such as plasmid DNA (Figure 1). Previously, we found that PAH-S.C. cells prepared with $5 \mathrm{mg} / \mathrm{mL}$ PAH were perfect for use in this system [12] and that $10^{6} \mathrm{cfu}$ of PAH-S.C. can absorb $5 \mu \mathrm{g}$ of plasmid DNA. The particle sizes and surface charges of S.C., PAH-S.C., or DNA-PAH-Salmonella prepared with $5 \mu \mathrm{g} / \mathrm{mL}$ of plasmid DNA were measured; and the results are listed in Table 1. Dynamic light scattering analysis demonstrated that the average size of the PAH-S.C. particles was increased compared with S.C. only. The surface charges of the S.C. and PAH-S.C. particles were measured as the zeta potential. The gradual increase in particle size and neutralization of the Salmonella surface charge suggested that the Salmonella were well coated with PAH. When the plasmid DNA was attached to the surface of the PAH-S.C., the particle size and surface charges of the DNA-PAH-S.C. were increased (Table 1). Meanwhile, the replication rates of S.C., PAH-S.C., or DNA-PAH-S.C. were measured to determine whether the PAH and plasmids coating the surface of Salmonella affected the physiology of the bacterium. When Salmonella were coated with plasmid and PAH, the growth curve of the Salmonella was slightly decreased compared with the control (Figure 2a). The growth rates of the tested groups were not significantly different from that of the S.C. group at $6 \mathrm{~h}$. We also examined the infective efficiency of DNA-PAH-S.C. cells and used gentamicin protection assays to measure the degree of infection of tumor cells by PAH-S.C. The bacterial invasion assay demonstrated that the invasion efficiency of DNA-PAH-S.C. slightly decreased with the plasmid and PAH coating (Figure 2b). Previously, we demonstrated that Salmonella are effective gene transfer vectors in vitro and in vivo [18]. Tumor cells infected with PAH-S.C. coated with EGFP plasmid (pTCY-EGFP) displayed significant fluorescence signals (Figure 3a). Furthermore, to test whether DNA-PAH-S.C. were able to transfer genes into tumor cells, luciferase expression was measured after infection with PAH-S.C. coated with a luciferase gene (Luc-PAH-S.C.) (Figure 3b). Our data supports the hypothesis that the DNA-PAH-S.C. retained the ability to infect tumor cells in vitro and transferred the gene to tumor cells. 
Figure 1. Schematic illustration of the strategy for the preparation of DNA-poly(allylamine hydrochloride) (PAH)-Salmonella.

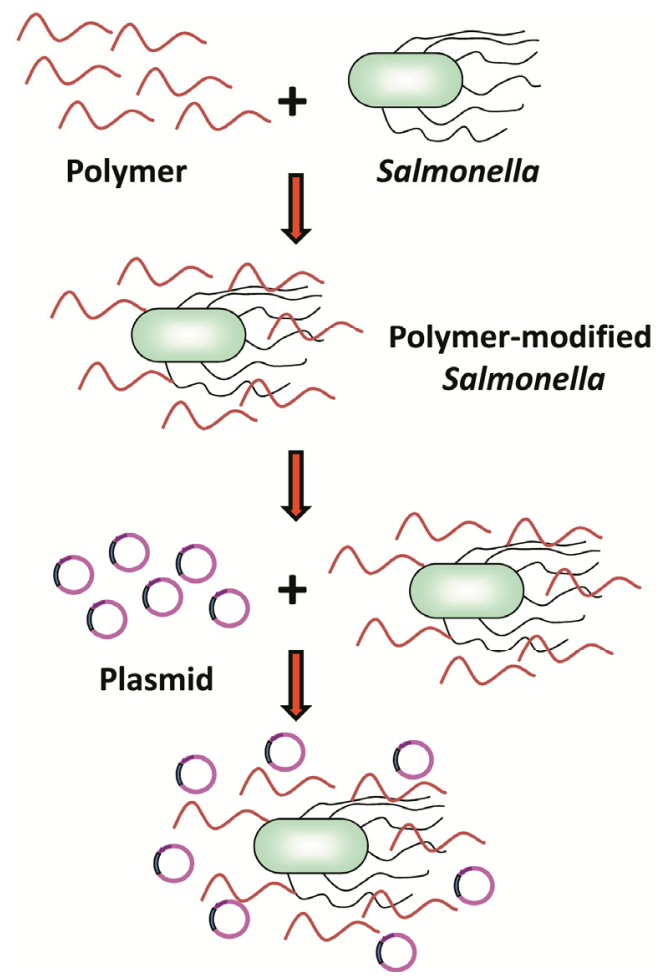

Table 1. Particle size and zeta potential values of Salmonella $(n=4)$.

\begin{tabular}{ccc}
\hline Bacteria & Particle size $(\mathbf{n m})$ & Zeta potential $(\mathbf{m V})$ \\
\hline Salmonella & $1068.25 \pm 80.52$ & $-13.8 \pm 1.60$ \\
PAH-Salmonella & $2279.25 \pm 415.58$ & $6.08 \pm 3.93$ \\
DNA-PAH-Salmonella & $4774.00 \pm 993.37$ & $-22 \pm 6.89$ \\
\hline
\end{tabular}

Figure 2. Replication and invasion activity of DNA-PAH-Salmonella (S.C.). (a) DNA-PAH-S.C. replication. The number of DNA-PAH-S.C. cells was determined $6 \mathrm{~h}$ post-incubation; and (b) The 4T1 cells were infected with $10^{6} \mathrm{cfu}$ of DNA-PAH-S.C., PAH-S.C., or S.C. cells. A gentamicin protection assay was used to examine these cells $9.5 \mathrm{~h}$ later. The data are reported as the means $\pm \operatorname{SD}(n=3)$.

a

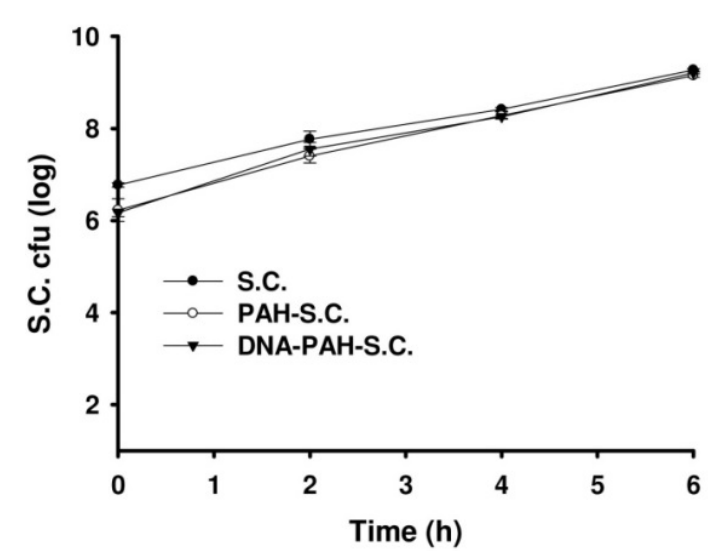

b

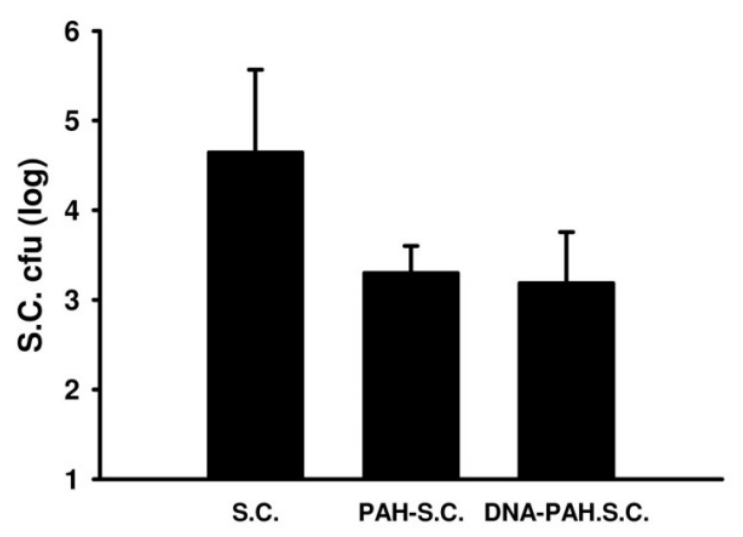


Figure 3. Transduction ability of DNA-PAH-S.C. (a) Cells transduced by Salmonella (S.C.) and coated with PAH and EGFP. EGFP was imaged using fluorescence microscopy, and cell nuclei were counterstained with 4',6-diamidino-2-phenylindole (DAPI); and (b) The effect of DNA-PAH-S.C. on gene transfer in vitro. 4T1 cells were infected with S.C. $\left(10^{6} \mathrm{cfu}\right)$, PAH-S.C. $\left(10^{6} \mathrm{cfu}\right)$, or Luc-PAH-S.C. $\left(10^{6} \mathrm{cfu}\right)$. Luciferase activities in these cells were measured $16 \mathrm{~h}$ later.

a

DAPI

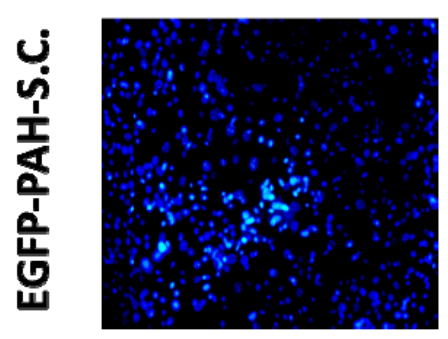

b

\section{EGFP}
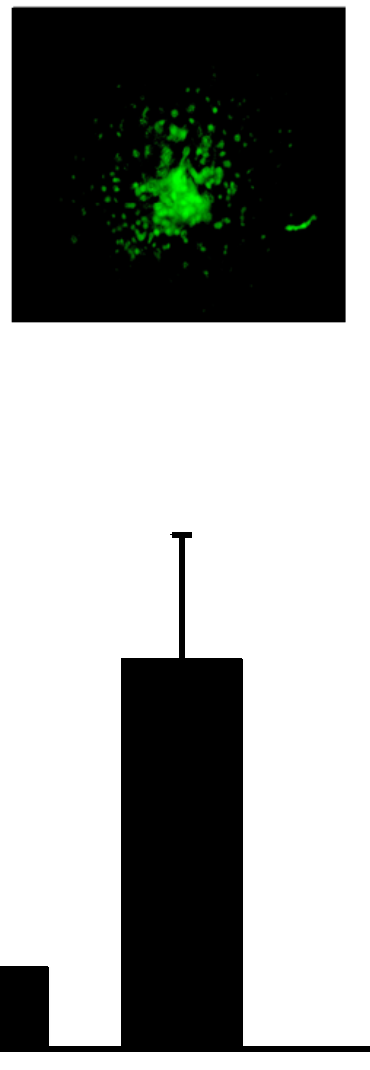

S.C. PAH-S.C.LUC-PAH-S.C.

\subsection{Tissue Distributions of DNA-PAH-S.C. in Mice}

To investigate the tissue distribution of DNA-PAH-S.C., tumor-bearing mice were i.v. injected with various modified S.C., and the amounts of Salmonella in the tumors, livers, and spleens were determined at day 10. As shown in Figure 4a, systemically administered S.C., PAH-S.C., or DNA-PAH-S.C. preferentially accumulated within the tumors at tumor-to-normal tissue accumulation ratios of 1000-10,000:1. Notably, the DNA-PAH-S.C. restored the tumor-targeting ability of Salmonella in mice. As shown in Figure 4a, 10 days after DNA-PAH-S.C. inoculation, the amounts of Salmonella in the tumors were approximately 4 orders of magnitude higher than those found in the spleen and liver. In addition, luciferase expression was readily observed at the tumor sites after Luc-PAH-S.C. inoculation (Figure 4b). Previously, we found that Salmonella PAH coating was diluted with each subsequent cell division, and the level of polymer coating continuously decreased during bacterial division. Our data support the hypothesis that the large size of DNA-PAH-S.C. retained the ability to 
infect tumor cells in vitro and in vivo (Figures 2-4). The DNA-PAH-S.C. displayed tumor-targeting efficiency and gene transfer ability in vivo.

Figure 4. Tumor-targeting potential and gene transfer of Salmonella (S.C.) coated with PAH and DNA. Mice were subcutaneously injected with tumor cells $\left(10^{6}\right)$. (a) Mice bearing 4T1 tumors ranging from 50 to $100 \mathrm{~mm}^{3}$ were injected i.v. with S.C., PAH-S.C., or DNA-PAH-S.C. $\left(2 \times 10^{6} \mathrm{cfu}\right)$ at day 7 , and the numbers of Salmonella cells in the tumors, livers, and spleens were determined at 10 days post-infection. The data are reported as the means $\pm \mathrm{SD}(n=4)$; and (b) The mice bearing 4T1 tumors were injected i.v. with $2 \times 10^{6}$ cfu of S.C., PAH-S.C., or Luc-PAH S.C.; and the expression levels of luciferase in tissues derived from the various modified, S.C.-treated mice at day 1 post-infection were determined using luciferase assays. The data are reported as the means $\pm \operatorname{SD}(n=4)$.

a

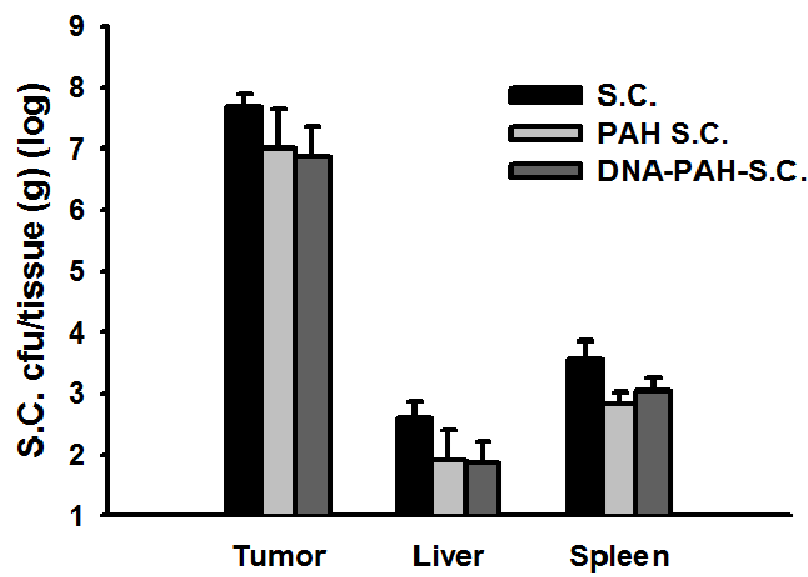

b

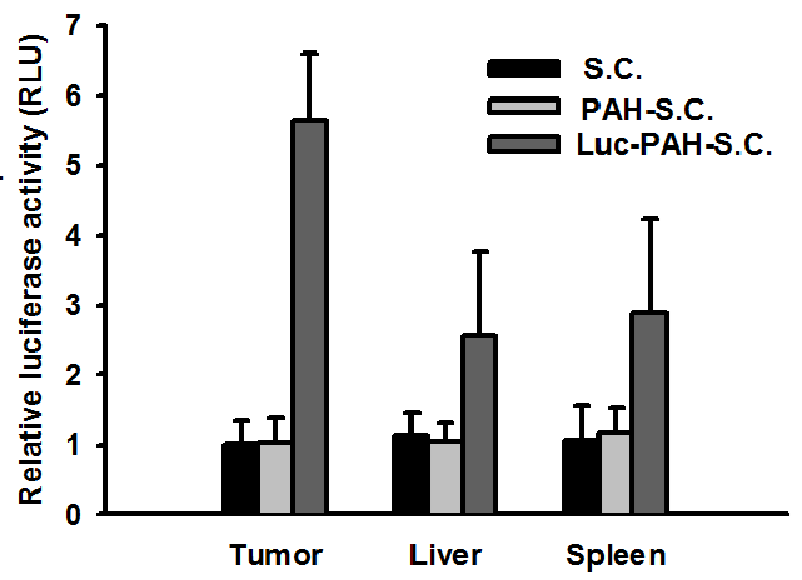

\subsection{Inhibition of Tumor Growth by DNA-PAH-Modified Salmonella}

Recently, connexin 43 (Cx43) has been identified as a tumor suppressor gene [19]. We also found that $\mathrm{Cx} 43$ plays an important antitumor role through $\mathrm{Cx} 43$ gap junction communication [20]. Herein, we used $C x 43$ as therapeutic gene and tested its antitumor activity by PAH-S.C.-coating the $C x 43$ gene (pcDNA-3.1-Cx43) (Cx43-PAH-S.C.). Figure 5a reveals that Cx43-PAH-S.C. treatment successfully delivered the $C x 43$ gene to tumor sites. $C x 43$ expression in the tumors of the mice treated with Cx43-PAH-S.C. was approximately 2.78-fold higher than those treated with PBS. Notably, the PAH-S.C. group also showed slightly increased Cx43 expression, and Salmonella-infection caused the cells to express Cx43 [10]. The antitumor effects of PAH-S.C. and Cx43-PAH-S.C. were evaluated in terms of tumor growth in mice bearing tumors. PAH-S.C. and Cx43-PAH-S.C. treatment both reduced tumor growth compared with PBS treatment in mice. Interestingly, PAH-S.C. treatment significantly reduced the tumor size by $22.23 \%$ compared with PBS treatment in mice (Figure $5 \mathrm{~b}$ ). The mean tumor volume for mice in the Cx43-PAH-S.C.-treated group decreased by $43.53 \%$ compared with those in the PAH-S.C.-treated group. Overall, Cx43-PAH-S.C. significantly suppressed tumor growth in mice. 
Figure 5. The antitumor effects of $\mathrm{Cx} 43-\mathrm{PAH}-\mathrm{S} . \mathrm{C}$. on mice. Mice bearing 4T1 tumors were injected i.v. with PAH-S.C. $\left(2 \times 10^{6} \mathrm{cfu}\right)$ or Cx43-PAH-S.C. $\left(2 \times 10^{6} \mathrm{cfu}\right)$ on day 7 . The vehicle control mice received PBS. (a) Cx43-PAH-S.C. induced $\mathrm{Cx} 43$ protein expression in tumors in vivo. 4T1-established tumors were treated with PBS, PAH-S.C., or Cx43-PAH-S.C. Three days later, the mice were killed, the tumors were collected, and the tumor lysates were analyzed for $\mathrm{Cx} 43$ expression using immunoblot analysis; and (b) Tumor volumes (mean $\pm \mathrm{SEM}, n=8$ ) from mice bearing 4T1 tumors were compared among the different treatment groups. ${ }^{*} p<0.05 ; * * p<0.01$.

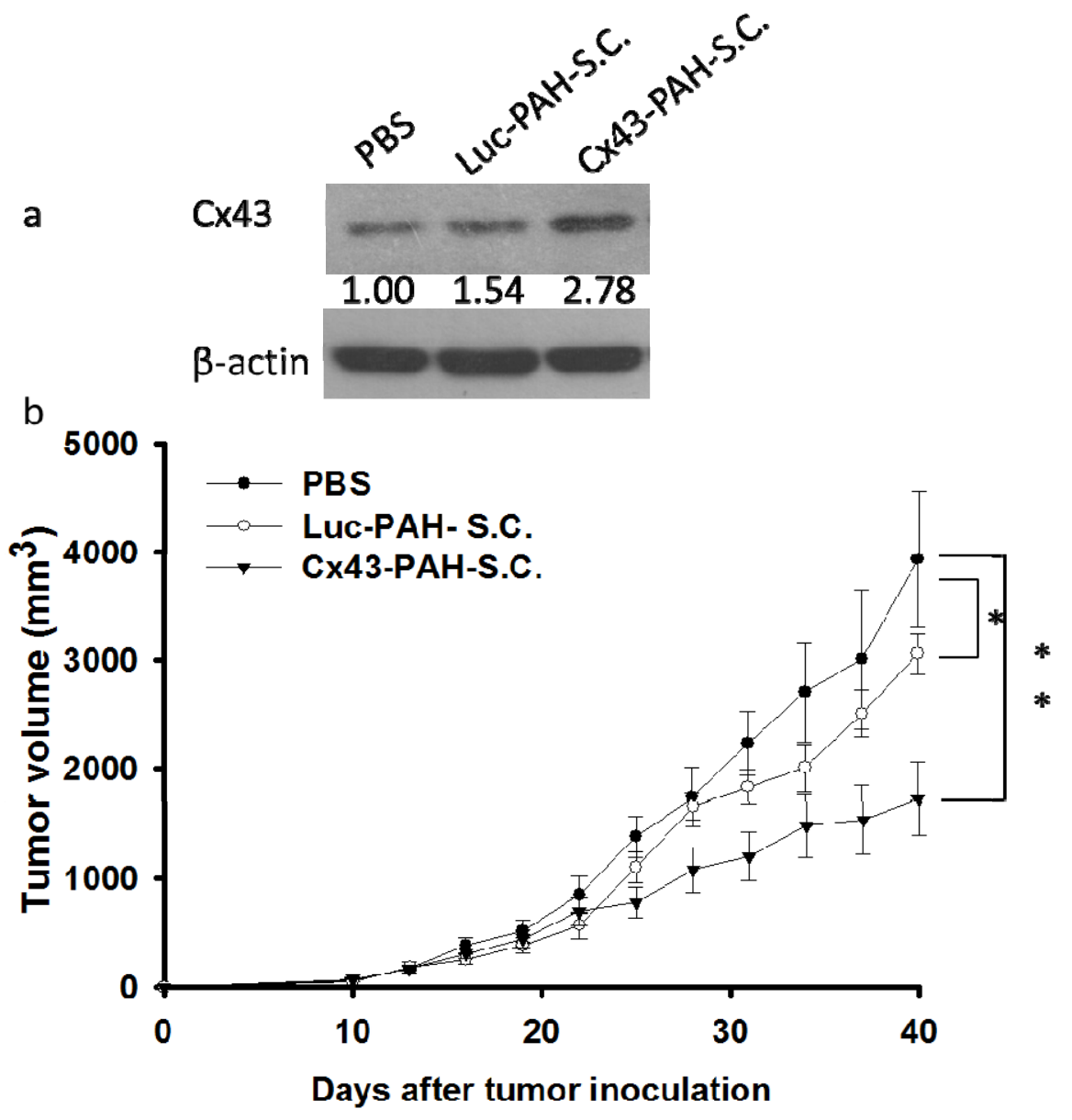

\section{Conclusions}

Herein, the encapsulation of Salmonella in polymer increases the gene transfer ability of Salmonella following in vivo delivery. The polymer-modified Salmonella delivered a gene to tumor sites and replicated in tumor sites, which are believed to stimulate non-specific antitumor immunity. These results help define the obstacles that it is necessary to overcome in order to deliver successful bacterial gene therapy.

\section{Acknowledgments}

This work was supported by grants from the National Science Council (NSC 101-2320-B-039-012-MY3, NSC 102-2621-B-035-001). 


\section{Author Contributions}

W.K.W. and C.Y.K. conceived and designed the experiments. Y.D.K. performed the experiments. C.H.L. analyzed the data. C.H.L. wrote the manuscript.

\section{Conflicts of Interest}

All of the authors have no conflict of interest.

\section{References}

1. Liu, F.; Zhang, L.; Hoffman, R.M.; Zhao, M. Vessel destruction by tumor-targeting Salmonella typhimurium A1-R is enhanced by high tumor vascularity. Cell. Cycle 2010, 9, 4518-4524.

2. Nagakura, C.; Hayashi, K.; Zhao, M.; Yamauchi, K.; Yamamoto, N.; Tsuchiya, H.; Tomita, K.; Bouvet, M.; Hoffman, R.M. Efficacy of a genetically-modified Salmonella typhimurium in an orthotopic human pancreatic cancer in nude mice. Anticancer Res. 2009, 29, 1873-1878.

3. Yam, C.; Zhao, M.; Hayashi, K.; Ma, H.; Kishimoto, H.; McElroy, M.; Bouvet, M.; Hoffman, R.M. Monotherapy with a tumor-targeting mutant of S. typhimurium inhibits liver metastasis in a mouse model of pancreatic cancer. J. Surg. Res. 2010, 164, 248-255.

4. Hayashi, K.; Zhao, M.; Yamauchi, K.; Yamamoto, N.; Tsuchiya, H.; Tomita, K.; Kishimoto, H.; Bouvet, M.; Hoffman, R.M. Systemic targeting of primary bone tumor and lung metastasis of high-grade osteosarcoma in nude mice with a tumor-selective strain of Salmonella typhimurium. Cell. Cycle 2009, 8, 870-875.

5. Kimura, H.; Zhang, L.; Zhao, M.; Hayashi, K.; Tsuchiya, H.; Tomita, K.; Bouvet, M.; Wessels, J.; Hoffman, R.M. Targeted therapy of spinal cord glioma with a genetically modified Salmonella typhimurium. Cell. Prolif. 2010, 43, 41-48.

6. Lee, C.H.; Wu, C.L.; Shiau, A.L. Endostatin gene therapy delivered by Salmonella choleraesuis in murine tumor models. J. Gene Med. 2004, 6, 1382-1393.

7. Zhao, M.; Yang, M.; Ma, H.; Li, X.; Tan, X.; Li, S.; Yang, Z.; Hoffman, R.M. Targeted therapy with a Salmonella typhimurium leucine-arginine auxotroph cures orthotopic human breast tumors in nude mice. Cancer Res. 2006, 66, 7647-752.

8. Zhao, M.; Yang, M.; Li, X.M.; Jiang, P.; Baranov, E.; Li, S.; Xu, M.; Penman, S.; Hoffman, R.M. Tumor-targeting bacterial therapy with amino acid auxotrophs of GFP-expressing Salmonella typhimurium. Proc. Natl. Acad. Sci. USA 2005, 102, 755-760.

9. Harris, T.J.; Green, J.J. Fung, P.W.; Langer, R.; Anderson, D.G.; Bhatia, S.N. Tissue-specific gene delivery via nanoparticle coating. Biomaterials 2010, 31, 998-1006.

10. Chang, W.W.; Lai, C.H.; Chen, M.C.; Liu, C.F.; Kuan, Y.D.; Lin, S.T.; Lee, C.H. Salmonella enhance chemosensitivity in tumor through connexin 43 upregulation. Int. J. Cancer 2013, 133, 1926-1935.

11. Chang, W.W.; Kuan, Y.D.; Chen, M.C.; Lin, S.T.; Lee, C.H. Tracking of mouse breast cancer stem-like cells with Salmonella. Exp. Biol. Med. 2012, 237, 1189-1196. 
12. Lee, C.H.; Lin, Y.H.; Hsieh, J.L.; Chen, M.C.; Kuo, W.L. A polymer coating applied to Salmonella prevents the binding of Salmonella-specific antibodies. Int. J. Cancer 2013, 132, 717-725.

13. Shiau, A.L.; Shen, Y.T.; Hsieh, J.L.; Wu, C.L.; Lee, C.H. Scutellaria barbata inhibits angiogenesis through downregulation of HIF-1 $\alpha$ in lung tumor. Environ. Toxicol. 2014, 29, 363-370.

14. Chang, W.W.; Liu, J.J.; Liu, C.F.; Liu, W.S.; Lim, Y.P.; Cheng, Y.J.; Lee, C.H. An extract of Rhodobacter sphaeroides reduces cisplatin-induced nephrotoxicity in mice. Toxins 2013, 5, 2353-2365.

15. ImageJ. Image Processing and Analysis in Java. Available online: http://rsbweb.nih.gov/ij/ (accessed on 6 September 2013).

16. Liu, W.S.; Kuan, Y.D.; Chiu, K.H.; Wang, W.K.; Chang, F.H.; Liu, C.H.; Lee, C.H. The extract of Rhodobacter sphaeroides inhibits melanogenesis through the MEK/ERK signaling pathway. Mar. Drugs 2013, 11, 1899-1908.

17. Lee, C.H.; Lin, S.T.; Liu, J.J.; Chang, W.W.; Hsieh, J.L.; Wang, W.K. Salmonella induce autophagy in melanoma by the downregulation of AKT/mTOR pathway. Gene Ther. 2014, 21, 309-316.

18. Lee, C.H.; Wu, C.L.; Chen, S.H.; Shiau, A.L. Humoral immune responses inhibit the antitumor activities mediated by Salmonella enterica serovar Choleraesuis. J. Immunother. 2009, 32, 376-388.

19. Ableser, M.J.; Penuela, S.; Lee, J.; Shao, Q.; Laird, D.W. Connexin43 reduces melanoma growth within a keratinocyte microenvironment and during tumorigenesis in vivo. J. Biol. Chem. 2014, 289, 1592-1603.

20. Cheng, Y.J.; Chang, M.Y.; Chang, W.W.; Wang, W.K.; Liu, C.F.; Lin, S.T.; Lee, C.H. Resveratrol enhances chemosensitivity in mouse melanoma model through connexin 43 upregulation. Environ. Toxicol. 2014, doi:10.1002/tox.21952.

(C) 2014 by the authors; licensee MDPI, Basel, Switzerland. This article is an open access article distributed under the terms and conditions of the Creative Commons Attribution license (http://creativecommons.org/licenses/by/3.0/). 